\title{
The pattern of northern hemisphere surface air temperature during prolonged periods of low solar output
}

\author{
Alexander Ruzmaikin, ${ }^{1}$ Joan Feynman, ${ }^{1}$ Xun Jiang, ${ }^{2}$ David C. Noone, ${ }^{2}$ Anne M. Waple, ${ }^{3}$ \\ and Yuk L. Yung ${ }^{2}$ \\ Received 11 March 2004; revised 6 April 2004; accepted 14 April 2004; published 16 June 2004
}

[1] We show that the reconstructed sensitivity of the sea level temperature to long term solar forcing in the Northern Hemisphere is in very good agreement with the empirical temperature pattern corresponding to changes of the North Annular Mode (NAM). This implies that long-term variations of solar output affect climate predominantly through the NAM that extends throughout the stratosphere and troposphere. INDEX TERMS: 1650 Global Change: Solar variability; 1620 Global Change: Climate dynamics (3309); 3309 Meteorology and Atmospheric Dynamics: Climatology (1620); 3344 Meteorology and Atmospheric Dynamics: Paleoclimatology; 3362 Meteorology and Atmospheric Dynamics: Stratosphere/ troposphere interactions; 7538 Solar Physics, Astrophysics, and Astronomy: Solar irradiance. Citation: Ruzmaikin, A., J. Feynman, X. Jiang, D. C. Noone, A. M. Waple, and Y. L. Yung (2004), The pattern of northern hemisphere surface air temperature during prolonged periods of low solar output, Geophys. Res. Lett., 31, L12201, doi:10.1029/2004GL019955.

\section{Introduction}

[2] Although anthropogenic greenhouse gases have been the major contributors to global warming during the last half of the 20th century, there is considerable evidence that solar variability played an important role in earlier climate history [Lean and Rind, 2001; Shindell et al., 2001]. One such period is from 1900 to the 1950 's, the early twentieth century [Cliver et al., 1998]. Other suggested periods occurred earlier: the Medieval Maximum (around 1200), the Spoerer Minimum (1450-1550), the Maunder Minimum (1645-1715), and the Dalton Minimum (around 1800 ), during which solar activity was enhanced or suppressed [Jirikowic and Damon, 1994]. The most studied period is the Maunder Minimum during which winter temperatures in Northern Europe were depressed. Regional mean winter temperatures in North America and Northern Europe were lower by $1-1.5^{\circ} \mathrm{C}$ [Pfister, 1995].

[3] Long-term changes in the solar output are independently observed in sunspots, geomagnetic activity, auroral appearances and in cosmogenic isotopes. Sunspots have been observed telescopically from the time of Galileo. Geomagnetic activity, driven by the solar wind, has been

\footnotetext{
${ }^{1}$ Jet Propulsion Laboratory, California Institute of Technology, Pasadena, California, USA.

${ }^{2}$ Department of Geological and Planetary Sciences, California Institute of Technology, Pasadena, California, USA. USA.

${ }^{3}$ STG, Inc., National Climatic Data Center, Asheville, North Carolina,
}

Copyright 2004 by the American Geophysical Union. 0094-8276/04/2004GL019955 measured since mid of 19th century. Scientifically useful records of auroral observations began in $450 \mathrm{AD}$ [Silverman, 1992]. ${ }^{14} C$ covers thousands of years until the 20th century when it became contaminated by anthropogenic carbon. ${ }^{10} \mathrm{Be}$ covering $10^{5}$ years shows the same behavior as ${ }^{14} \mathrm{C}$ but is not contaminated by anthropogenic isotopes [Beer, 2000]. When these data sets overlap, they all testify to the same changes: weak solar outputs at the beginnings of the 18th, 19th and 20th centuries and during the Maunder Minimum (16401720) and strong solar outputs during the 12th century.

[4] These low-frequency variations in the solar output affect the Earth's atmosphere. The strongest radiative effects are expected in the UV irradiance that causes temperature and ozone changes at the top of the stratosphere [Lean and Rind, 2001]. The stratosphere and the troposphere are connected dynamically through a major mode of atmospheric variability, called the North Annular Mode (NAM) in the Northern Hemisphere and the South Annual Mode (SAM) in the Southern Hemisphere. The NAM currently accounts for an average of $22 \%$ of the wintertime variance of the sea level pressure in the Northern Hemisphere at sea level [Thompson and Wallace, 1998]. A robust relationship between solar cycle variations, proxied by the $10.7 \mathrm{~cm}$ solar radiation, and the NAM has been found for the current epoch 1958-1997 using the NCEP Reanalysis data [Ruzmaikin and Feynman, 2002]. In particular the mean index of NAM, defined as the principal component (PC) of the first Empirical Orthogonal Function (EOF) of geopotential height anomalies, was found to be systematically more negative (corresponding to a weaker polar jet) all the way through the stratosphere and troposphere during low solar activity. This "propagation" through the stratosphere into the troposphere without damping distinguishes this effect from the well-known Van Loon-Labitzky effect of UV on the upper stratosphere during West QBO in late winter, which damps out at the tropopause and so cannot effect the lower troposphere temperature. [These results are not significantly changed by the omission of data from the two years after each of the Al Agung, El Chicon and Pinatubo volcanic eruptions.] The tendency for the atmosphere to be in the low NAM state was shown in GCM modeling. The modeling has also demonstrated that regional climate impacts of volcanic and solar forcing are distinctively different [Shindell et al., 2003]. In particular, in contrast to the response to solar forcing the long-term (decadal and longer) response to volcanic eruptions is relatively weak due to opposing radiative and dynamic effects.

[5] Here we show that the reconstructed sensitivity of the sea level temperature to long term solar forcing in the Northern Hemisphere [Waple et al., 2002] is in a very good agreement with the temperature pattern corresponding to a 

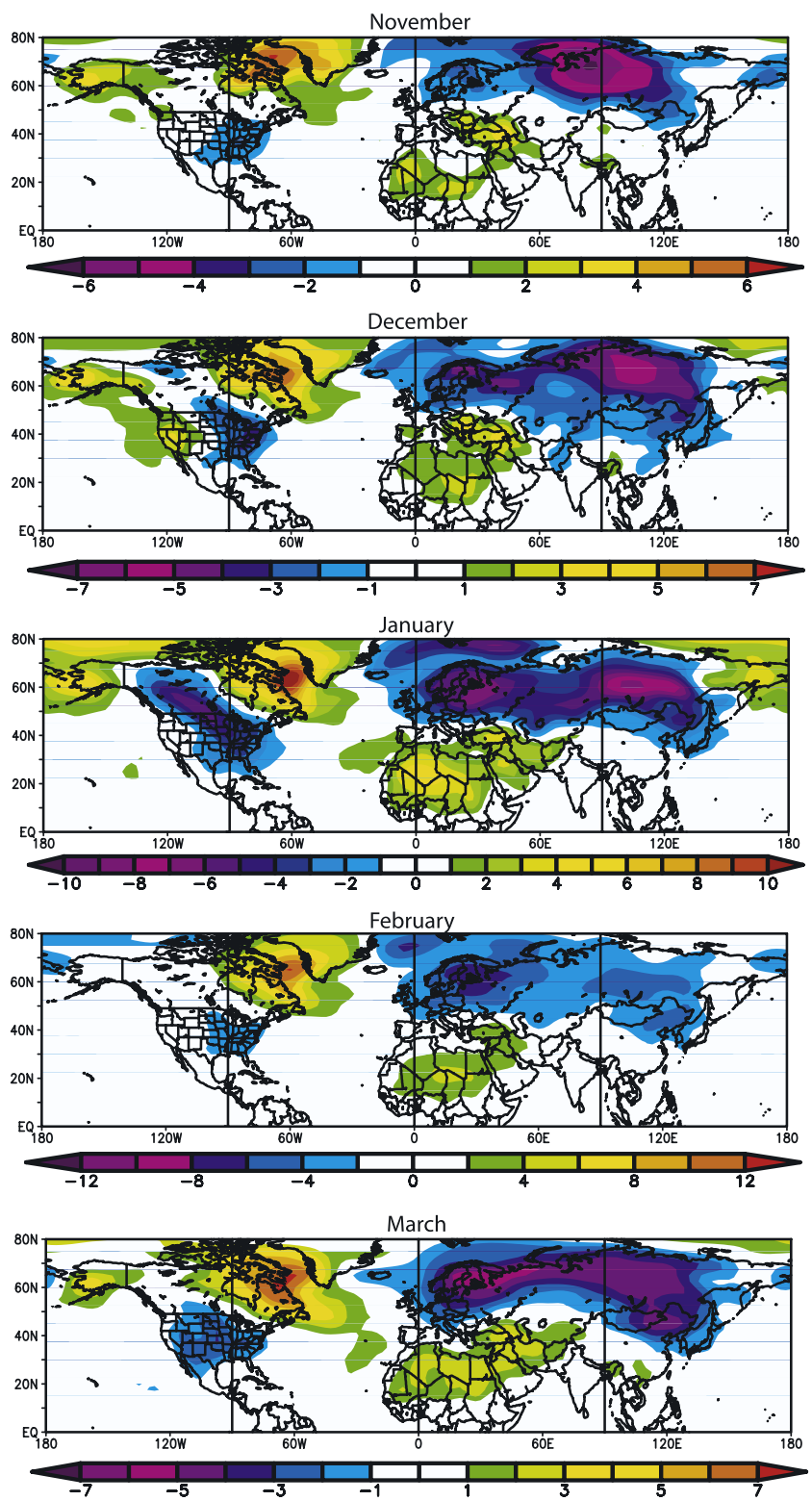

Figure 1. The monthly composite differences of the NCEP Reanalysis of air temperature data at $1000 \mathrm{hPa}$ between years of high and low NAM index for the months of November through March (for example 1950, 52, 158, 61, 95, 96, 2001, 2002 for low negative indices, and 1951, 53, $54,57,90,94,98,99$ for positive NAM indices in December).

decrease in the NAM index. This implies that long-term variations of solar output influence climate of the extratropical Northern Hemisphere predominantly through the NAM and the solar effects on the higher modes are less important.

[6] To demonstrate the role of the NAM in the atmospheric response to solar variability we use NCEP Reanalysis monthly mean data to generate data sets in which the NAM dominates over all other contributions to the potential height anomalies (Section 2). We then find the corresponding temperature patterns for high and low NAM. In Section 3 the differences in these temperature patterns are compared with the temperature sensitivity to long-term solar output changes. The results are discussed in Section 4.

\section{Temperature Changes Associated With NAM Changes}

[7] Since the EOF's are orthogonal functions, the observed geopotential height pattern at any time can be expressed as a superposition of its EOFs, i.e., as $\sum_{n=1}^{\infty} P C_{n}(t) E O F_{n}(x, y)$, where $P C_{n}(t)$ is the principle component of the $E O F_{n}$ at time $t$. To isolate the temperature pattern due to the NAM we first choose months, marked $t_{1}$, $t_{2}, \ldots, t_{N}$, for which the $P C_{1}$, called the NAM index, has high positive values. Next we form a composite of these months. Since the $P C_{1}$ 's are all large and positive they add constructively. However the $P C_{n}$ with $n>1$ can have either sign and any value and tend to cancel each other in the composite. Hence $\left(P C_{1}\left(t_{1}\right)+P C_{1}\left(t_{2}\right)+\ldots\right) \gg\left(P C_{n}\left(t_{1}\right)+\right.$ $\left.P C_{n}\left(t_{2}\right)+\ldots\right)$ for any $n>1$. In practice, we find that a composite of monthly data from about 10 years give a $E O F_{1}$ pattern that is consistent from month to month. We then generate the temperature pattern $\left(T_{P}\right)$ for this composite. Note that the temperature pattern corresponding to the first EOF of geopotential heights does not necessarily coincide with the first EOF of the temperature. Also note that the pattern represents the NAM contribution to the temperature even when the NAM index is small compared with the indices of higher EOF's.

[8] The procedure is then repeated using months with the most negative NAM to find the corresponding temperature pattern $T_{N}$. The change in temperature pattern $\Delta T=T_{N}-T_{P}$ is due to the change of the atmosphere from the state with the large mean negative NAM index to the state with the large mean positive index.

[9] One possible source of error in this procedure comes from global warming. The NCEP Reanalysis data is a reconstruction of the observations and therefore contains all of the variability, including the systematic global warming caused by greenhouse gasses. The global warming is not randomly distributed, hence it would not be automatically suppressed in a composite. To examine this effect we generated the difference in composites of months for the same range of NAM indices before 1970 with years of NAM indices after 1990. The resulting pattern showed a narrow circumpolar warming over the last 50 years. Since there is no evidence for a systematic long-term increase in the solar output during this time [Arge et al., 2002] it represents the part of the global warming over the last 50 years, which is not due to solar forcing. The contamination to the composite NAMs due to this global warming is reduced as far as possible by choosing the years that make up the composites in such a way that each year chosen for the high NAM index data has a close partner year in the low NAM index data. With such a selection the contribution of global warming is minimized when the low NAM temperature data is subtracted from the high NAM data to find $\Delta T$.

[10] Our results for each of the winter months are shown in Figure 1. Altogether 96 months of data were used to generate these $\Delta T$ temperature patterns. The differences between the patterns reflect a combination of true seasonal variations and uncertainties in the data analysis due to any contribution from residual higher EOFs. The patterns have 


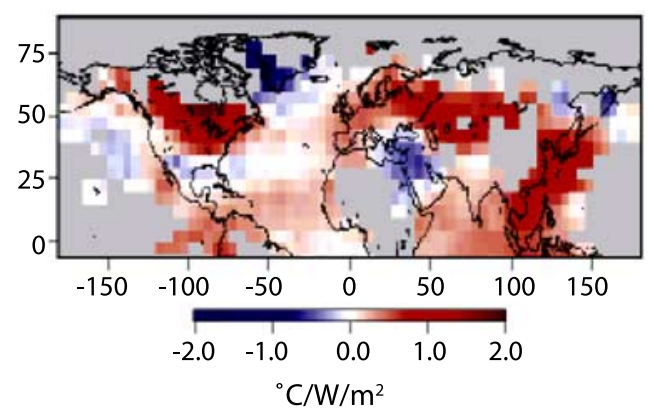

Figure 2. Sensitivity pattern of surface temperature reconstructions against the Lean et al. [1995] total solar irradiance reconstruction for the period 1650-1850 with time lag of 10 years, truncated for the Northern Hemisphere [Waple et al., 2002]. The gray color marks areas with insufficient data. Note that negative sign in sensitivity implies positive temperature anomaly during periods of low solar output.

important features in common. Siberia and Northern Europe experience a cooling, the extent of which changes as we go from fall toward spring. For example, the cooling region is mostly confined to Siberia in November but it spans the entire Eurasian continent in February. All months also show cooling in eastern North America, stretching into Canada in February. In contrast, for all months there is a strong heating in the area of Greenland, which for some months stretches to Northern Alaska. A less intense warming pattern also appears over North Africa and the Middle East.

[11] Note that we could not generate the NAM temperature patterns using the summer data. The reason is that the NAM index is much smaller in summer so that the higher EOF's are not sufficiently suppressed in the composite.

\section{Comparison With the Temperature Sensitivity to Solar Irradiance}

[12] Waple et al. [2002] reconstructed the pattern of correlations between sensitivity of empirically derived surface temperatures to solar irradiance variations for the period 1650 to 1850 . They show the regional sensitivity of the temperature to the solar irradiance in the period band exceeding 40 years. The temperature changes due to NAM variability found above and the Waple et al. map are in remarkably good agreement. For low solar output both the NAM temperature pattern and the temperature sensitivity to solar forcing (Figure 2) show cooling stretching across Europe through Siberia and even encompassing Japan to some extent. North America also experiences cooling south of Hudson Bay. In contrast, during these periods, the west coast of Greenland experiences strong heating in both datasets, and there is a less strong heating in the Middle East. The cooling in Northern Africa seen in our NAM patterns could not be verified because of lack of data for the temperature reconstruction. Thus the major part of the Northern Hemisphere temperature changes driven by the Sun can be attributed to the effect of solar changes on the NAM mode of atmospheric variability. This remarkable result appears particularly interesting when we take into consideration that annual averages used to generate Figure 2 include tree-ring data, a summer indicator associated with small NAM indices. In accord with our results, the summer sea level NAM index reconstructed from the tree-ring data is negative during the Maunder Minimum [D'Arrigo et al., 2003].

[13] The magnitude of the temperature changes believed to have taken place during the Maunder Minimum remains uncertain. A linear scaling of the changes in the NAM index from solar maximum to solar minimum found in earlier work [Ruzmaikin and Feynman, 2002] yields a factor of two larger change in NAM during the Maunder Minimum. This implies a change in Northern Europe temperature of about 1 degree. The global mean temperature anomaly is much less than that because of the averaging over the heating and cooling regions. This agrees with the Mann et al. [1998] reconstruction that shows the global mean temperature during the Maunder Minimum to be only a fraction of a degree cooler than the periods of higher solar output before and after it. However, the relationship between the temperature and the NAM is non-linear and more study is needed to evaluate the magnitude of the change.

\section{Discussion}

[14] Because the NAM is influenced by the interaction between planetary waves and zonal-mean zonal wind [Thompson and Wallace, 1998] it is important to check to what degree the patterns of variability found above are robust to changes to this interaction. A major cause of stationary planetary waves is the surface land/sea thermal contrast, which depends on the ocean-air coupling. We carried out two 10-year climate simulations using the National Center for Atmospheric Research Community Climate Model version 3 (CCM3) [Kiehl et al., 1998]. The model was configured to have a horizontal resolution of $2.8^{\circ} \times 2.8^{\circ}$ and 18 vertical levels. In the simulations, both the atmospheric composition and external forcing were fixed. In the first simulation, the ocean surface conditions (temperature and sea ice distribution) were prescribed from a 12-month climatology derived from satellite observations. In the second simulation, ocean temperature is computed interactively using a simple thermodynamic model of the mixed layer. Heat transport to and from the deep ocean is prescribed such that the predicted ocean surface temperature resembles observations. By allowing the ocean to have a finite heat capacity, local energy exchange provides an additional (negative) feedback for the surface exchange. Only in the second simulation (interactive ocean) we find general agreement between the simulated and the empirical patterns. This important role of the ocean was also indicated by Waple et al. [2002].

[15] To further confirm the physical processes involved, we investigated the wave (EP) flux transport during the periods used in the analysis described in Section 2. GCM modeling has previously shown [Limpasuvan and Hartmann, 2000] that a negative NAM is associated with increased heat transport to the polar regions. We found that this is also the case for the sub-sample of NCEP Reanalysis data used here. The NAM apparently involves a coupling of the planetary waves and the temperature into a non-linear dissipative system, with a damping time $\alpha^{-1} \approx 30$ days in the low stratosphere. This is supported by the observations that the March polar temperature is strongly correlated with 
the January-February EP flux in the $100-400 \mathrm{hPa}$ and $45^{\circ}-75^{\circ} \mathrm{N}$ [Newman, et al., 2001]. The NAM is obviously subjected to a number of forcings, from powerful synoptic activity in the troposphere to QBO, volcanic and demonstrated here solar variability forcing in the stratosphere.

[16] In summary: the strong similarity of patterns of sensitivity to solar forcing with that corresponding to changing the isolated NAM index implies that variations of the solar output primarily affect the North Annular Mode of atmospheric variability. This also lends additional credence to the mechanism of solar influence on climate driven by the changes of the UV irradiance in the stratosphere.

[17] Acknowledgments. The images shown in Figure 1 are generated by using the NOAA- CIRES Climate Diagnostics Center web site. This work was supported in part by the Jet Propulsion Laboratory of the California Institute of Technology, under a contract with NASA, and by the NASA LWS grant to the California Institute of Technology.

\section{References}

Arge, C. N., E. Hildner, V. J. Pizzo, and J. W. Harvey (2002), Two solar cycles of non-increasing magnetic flux, J. Geophys. Res., 107(A10), 1319, doi:10.1029/2001JA000503.

Beer, J. (2000), Long-term indirect indices of solar variability, Space Sci. Rev., 11, 53-66.

Cliver, E. W., V. Boriakov, and J. Feynman (1998), Solar variability and climate change: Geomagnetic aa index and global surface temperature, Geophys. Res. Lett., 25(7), 1035-1038.

D’Arrigo, R. D., E. R. Cook, M. E. Mann, and G. C. Jacoby (2003), Treering reconstruction of temperature and sea-level pressure variability associated with the warm-season Artic Oscillation since AD 1650, Geophys. Res. Lett., 30(11), 1549, doi:10.1029/2003GL017250.

Jirikowic, J. L., and P. Damon (1994), The medieval solar maximum, Clim. Change, 26, 305-316.

Kiehl, J. T., J. J. Hack, G. B. Bonan, B. A. Boville, D. L. Williamson, and P. J. Rasch (1998), The National Center for Atmospheric Research Community Climate Model: CCM3, J. Clim., 11(6), 1131-1149.

Lean, J., O. R. White, and A. Skumanich (1995), On the solar ultraviolet spectral irradiance during the Maunder Minimum, Global Biogeochem, Cycles, 9, $171-182$.
Lean, J., and D. Rind (2001), Earth's response to a variable Sun, Science, 292, 234-236.

Limpasuvan, V., and D. L. Hartmann (2000), Wave-maintained annular modes of climate variability, J. Clim., 13, 4414-4429.

Newman, P., E. Nash, and J. Rosenfield (2001), What controls the temperature of the Arctic stratosphere during the spring?, J. Geophys. Res., 106(D17), 19,999-20,010.

Mann, M. E., R. S. Bradley, and M. K. Hughes (1998), Global-scale temperature patterns and climate forcing over the past six centuries, Nature, 392, 779-787.

Pfister, C. (1995), Monthly temperature and precipitation in central Europe from 1525-1979: Quantifying documentary evidence on weather and its effects, in Climate Since 1500, edited by R. S. Bradley and P. D. Jones, pp. 118-142, Routkedge, London.

Ruzmaikin, A., and J. Feynman (2002), Solar influence on major mode of atmospheric variability, J. Geophys. Res., 107(D14), 4209, doi:10.1029/ 2001JD001239.

Shindell, D. T., G. A. Schmidt, M. Mann, D. Rind, and A. Wapple (2001), Solar forcing of regional climate during the Maunder Minimum, Science, 294, 2149-2152.

Shindell, D. T., G. A. Schmidt, R. L. Miller, and M. E. Mann (2003), Volcanic and solar forcing of climate change during the preindustrial era, J. Climate, 16, 4094-4107.

Silverman, S. (1992), Secular variation of the aurora for the past 500 years, Rev. Geophys., 30, 333-351.

Thompson, D. W. J., and J. M. Wallace (1998), The arctic oscillation signature in the wintertime geopotential height and temperature fields, Geophys. Res. Lett., 25(9), 1297-1300.

Waple, A. M., M. E. Mann, and R. S. Bradley (2002), Long-term patterns of solar irradiance forcing in model experiments and proxy based surface temperature reconstructions, Climate Dyn, 18, 563-578.

A. Ruzmaikin and J. Feynman, Jet Propulsion Laboratory, 4800 Oak Grove Dr., Pasadena, CA 91109, USA. (aruzmaik@pop.jpl.nasa.gov; joan.feynman@jpl.nasa.gov)

X. Jiang, D. C. Noone, and Y. L. Yung, California Institute of Technology, California Blvd., Pasadena, CA 91125, USA. (xun@gps. caltech.edu; dcn@gps.caltech.edu; yly@gps.caltech.edu)

A. M. Waple, STG, Inc., Government Contractor, National Climatic Data Center, 151 Patton Ave., Asheville, NC 28801, USA. (anne.waple@noaa. gov) 